\title{
Functional Impairments Post Breast Cancer Surgery: A Correlational Study
}

\author{
Prabhjot Gambhir \\ Max Super Specialty Hospital, Saket, New Delhi, India
}

\begin{abstract}
Physical impairments in upper limb following breast cancer surgery and/or radiation therapy have been documented in the literature. However, the extent of functional limitations in daily tasks has not been studied to a large extent. Among the measures studied for functional performance are upper extremity functional index (UEFI), Disabilities of the arm, shoulder, and hand (DASH) and International Classification of Functioning (ICF) corset for breast cancer. The functional limitations may be attributed to shoulder joint restrictions, lymphedema and sensory impairments. Thus, a correlational study was designed to learn the degree to which these questionnaires can measure the limitations in activities of daily living in individuals who have undergone breast cancer surgery.
\end{abstract}

Keywords: UEFI, ICF corset for breast cancer, DASH questionnaire, functional limitations, breast cancer.

\section{Introduction}

Traditionally, function has been viewed as interrelated area of physical performance, such as muscular strength, range of motion, and cardiopulmonary endurance. Within International Classification of Functioning (ICF) framework, function is defined as the interaction between an individual, their health condition, and the social and personal context in which they live. In the context of breast cancer, morbidity associated with the disease and its treatments can lead to impairments in physiological, psychological, or behavioural attributes potentially leading to limitations in ability to execute desired tasks and participation in social demands.

Post- treatment impairments in upper limb and upper body structure and function consist of upper limb edema, decreased shoulder mobility, neural tissue injuries causing sensory and motor dysfunction and pain. Both radiotherapy and surgery are associated with a range of long-term treatment-related morbidities $(1,2)$. Both impaired shoulder movements and lymphedema of the upper limb are more common after mastectomy than after a breast conservation surgery (3).

Muscle weakness of the upper limb has been found among $18-23 \%$ of patients $(4,5)$. The weakness can also progress and lead to loss of hand function. Tasmuth et.al found that chronic pain had a significant correlation with other posttreatment symptoms, such as edema, paresthesia, strange sensations and phantom pain (6). The incidence of numbness varies from $29 \%$ to $81 \%$ among breast cancer patients.

Gosselink et.al reported that MRM (Modified Radical Mastectomy) patients suffered greater functional limitations, concerning the Activities of Daily Living (ADL) of the upper limb than did BCS (Breast Conservation Surgery) patients (2). Studies show that patients with arm swelling experienced considerable activity limitations in home and work environments. Limited shoulder movements and numbness seem to increase self-assessed activity limitation.

In contrast to impairments in body functions and structures, little is known about activity limitations and participation restrictions and there is therefore an apparent need for such studies. Little is known of how the operation type affects activity limitations and participation restrictions. Karki et.al (7) found that activities most limited after breast cancer operation were lifting, carrying, and reaching out.

The current study was devised with the aim of assessing functional impairments associated with upper limb related ADL in females who had undergone breast cancer surgery and radiation therapy resulting in restriction in arm movements and lymphedema.

\section{Methodology}

Female subjects were selected as a convenient sample on an outpatient basis in the department of rehabilitation referred by oncologists for shoulder restriction and lymphedema after breast cancer surgery and radiation therapy. Demographic data was collected and the patients' were assessed for functional limitations on the following outcome measures:

1) Upper Extremity Functional Index (UEFI)

2) It assesses upper extremity functional performance. It is a 20 -item questionnaire rated on a 5-point likert scale $(0$ 4). The maximum score is 80 . Higher scores signify better functional performance. It has been recognized as a valid and sensitive to changes in breast cancer population (8).

3) Disabilities of the Arm, Shoulder, and Hand (DASH) questionnaire

4) It is a 30-item questionnaire rated on a 5-point likert scale (1-5) where higher scores are representative of greater difficulty in functional areas. After scoring all 30 items, a dash disability/symptom score is calculated (maximum score 100).

5) Activity and participation domain of International Classification of Functioning (ICF) breast cancer corset

6) The corset contains 5 areas in this domain viz. general tasks and demands, mobility, domestic life, interpersonal interactions and relationships, and major life areas. A total of 34 subcomponents are recognized under these 5 areas and scored on a 5-point likert scale viz. qualifiers where higher the score, greater the extent of functional limitations. 


\section{International Journal of Science and Research (IJSR) \\ ISSN (Online): 2319-7064}

Index Copernicus Value (2013): 6.14 | Impact Factor (2014): 5.611

7) Other domains of ICF viz. body structure, body function, and environmental domain were excluded to correlate functional performance with DASH and UEFI.

The scores were recorded for all 3 sets of measures and correlational statistics were conducted.

\section{Results \& Discussion}

The subjects were 40 females with an average age of 52.96 years who had undergone unilateral breast cancer surgery along with radiation therapy. All females were found to have affected side (23 left, 17 right) shoulder range of motion restrictions and lymphedema (grade 1 or 2) resulting in inability to participate fully in daily functional activities. None of the subjects had a history of shoulder restriction prior to surgery or of pain on the affected side.

The statistical analysis to calculate correlation was done using Pearson's correlation coefficient calculator.

Table 1: Mean scores of UEFI, DASH and ICF corset activities and participation domain

\begin{tabular}{|c|c|}
\hline Variable & Mean score \\
\hline Age (in years) & 52.93 \\
\hline UEFI & 71.43 \\
\hline DASH & 14.01 \\
\hline ICF (activities and participation & 22 \\
\hline
\end{tabular}

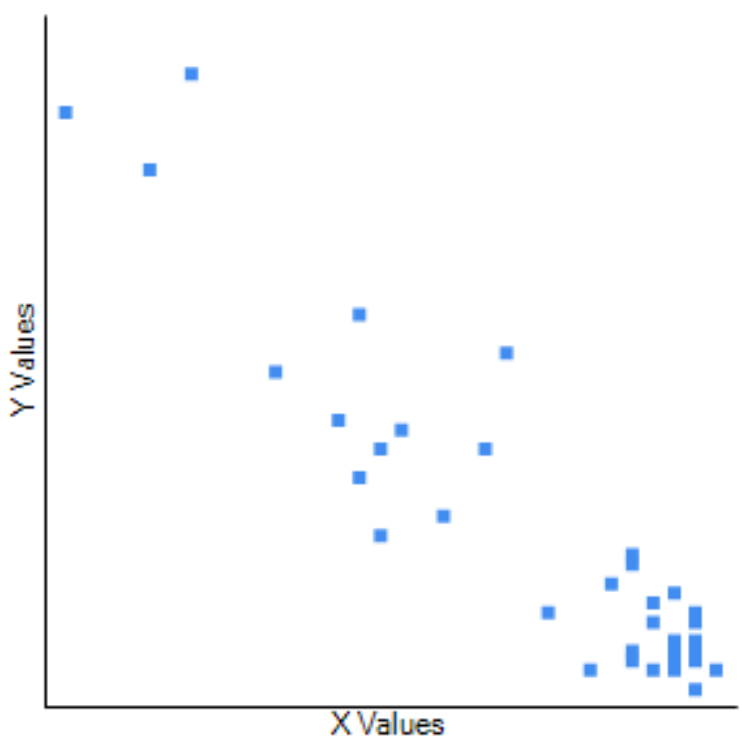

Figure 1: Correlation between UEFI scores and DASH scores

A strong negative correlation coefficient of - 0.9276 (r) was found between the UEFI and DASH scores. This signifies that the higher the UEFI scores, the lower will be the scores for DASH. UEFI represents functional index where higher scores mean better functional performance and low level of disability as shown by low DASH scores for upper limb.

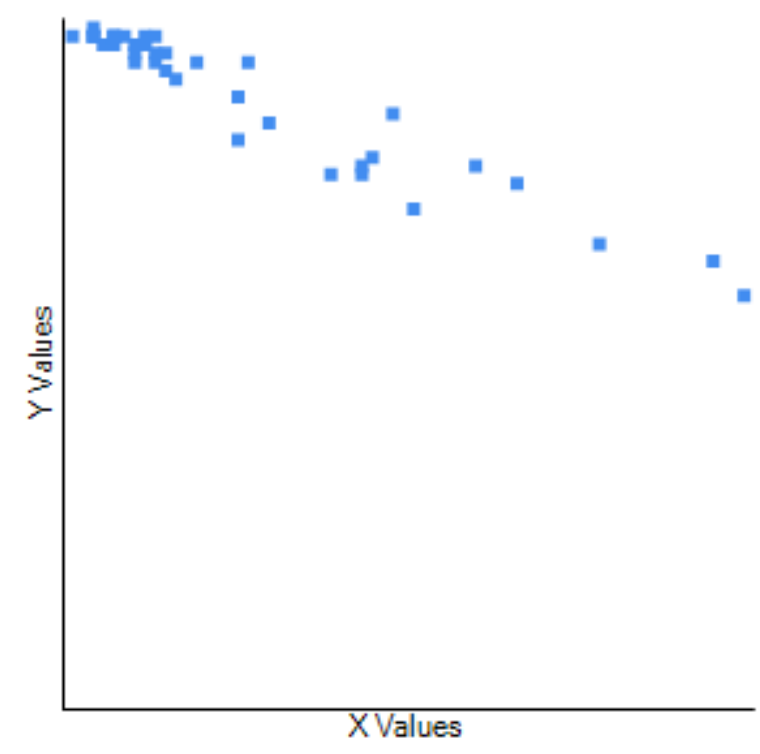

Figure 2: correlation between UEFI and ICF corset for activities and participation domain

The correlation between UEFI and ICF scores was also negative $(r=-0.9626)$ as ICF higher scores are significant of a greater dependence in daily activities. Therefore, a strong correlation shows that both variables are sensitive to assessing functional performance of breast cancer patients. However, improvement in functional performance is shown either by high UEFI scores or low ICF corset scores.

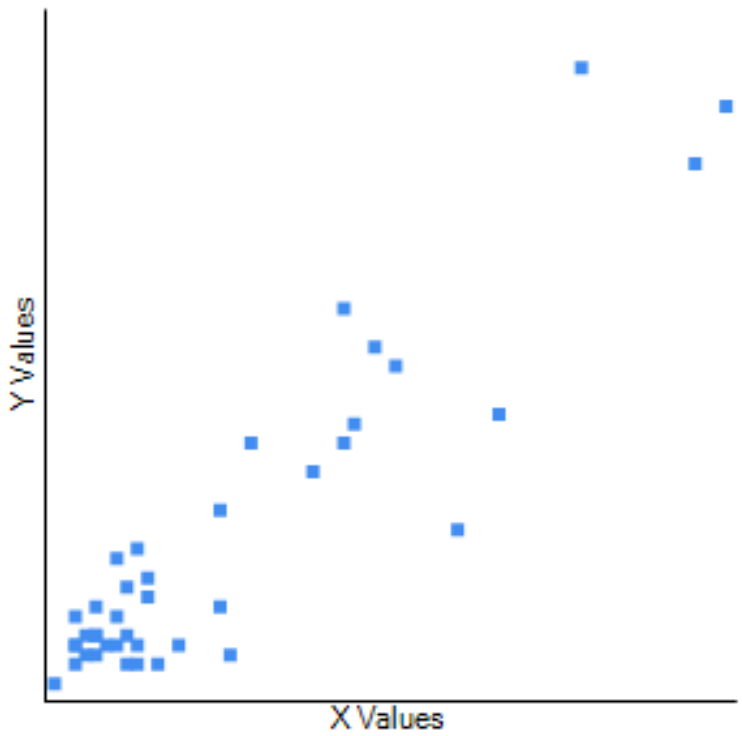

Figure 3: correlation between DASH and ICF activities and participation domain

A very strong positive correlation was found between DASH and ICF scores $(r=0.9175)$. Both sets of questionnaires assess an individual on daily activities along with social and household work related skills.

Lately, the number of survivors of breast cancer has increased because of advances in early detection and treatment. Short recovery times and the best possible functioning of patients after breast cancer operations should be the key interests of rehabilitation. 


\section{International Journal of Science and Research (IJSR) \\ ISSN (Online): 2319-7064 \\ Index Copernicus Value (2013): 6.14 | Impact Factor (2014): 5.611}

To ensure better functioning of individuals who have undergone breast cancer surgery, it is mandatory to have a valid and reliable measure to assess the functional performance. Upper limb impairments post surgery and radiation therapies have been documented throughout literature $(9,10,11)$. A key aspect in the development of rehabilitation approaches to improve function in breast cancer survivors is identifying measurement tools that can capture functional limitations to inform treatment decisions and rehabilitation programming. The measures need to be sensitive to the unique issues of breast cancer survivors and responsive to changes in the patients' status.

In the current study, a strong positive correlation was found between DASH and ICF corset for breast cancer (activities and participation domain) (Fig.1). Their scores show the extent of disability or dependence an individual faces in his daily functioning along with social skills. Also, a strong negative correlation was found between UEFI and DASH as well as UEFI and ICF (Fig. 2 and 3). UEFI is a measure of improving functional performance in daily activities related to upper limb.

It has been noted that women with lymphedema had greater limitation in upper extremity activities than those without a history of lymphedema (9). The females included in the present study had history of lymphedema as well as shoulder restriction. As statistically significant correlates were found between the 3 outcome measures used in this study, they all can provide a good measure of improvement noted after rehabilitation programs have been administered for better functioning of an individual in daily activities.

\section{Future Scope}

This study was conducted on a small sample of breast cancer population, thus the results can be further assessed on a larger population for outcome measures to be used in breast cancer patients for functional performance. Also, rehabilitation interventions can be studied based on these outcome measures to record improvement over a period of time.

\section{References}

[1] Rietman J, Djikstra P, Debreczeni R, Geertzen J, et.al. Impairments, disabilities and health - related quality of life after treatment for breast cancer: a follow-up study 2.7 years after surgery. Disability and Rehabilitation, 2004, Volume 26, pp. 78-84.

[2] Gosselink R, Rouffaer L, Vanhelden P, Piot W, et.al. Recovery of upper limb function after axillary dissection. J Surg Oncol, 2008, Volume 83, pp. 204211.

[3] Deutsch M, Flickinger J. Shoulder and arm problems after radiotherapy for primary breast cancer. Am J Clin Oncol, 2001, Volume 24, pp. 172-176.

[4] Kakuda J, Stuntz M, Trivedi V, Klein S, et.al. Objective assessment of axillary morbidity in breast cancer treatment. Am Surg, 1999, Volume 65, pp. 995-998.

[5] Johansen J, Overgaard J, Blichert-Toft M, Overgaard M. Treatment morbidity associated with the management of the axilla in breast- conserving therapy. Acta Oncol, 2000, Volume 39, pp. 347-354.

[6] Tasmuth T, Blomqvist C, Kalso E. Chronic posttreatment symptoms in patients with breast cancer operated in different surgical units. Euro J Surg Oncol, 1999, Volume 25, pp. 38-43.

[7] Karki A, Simonen R, Malkia E, Selfe J. Impairments, activity limitations and participation restrictions 6 and 12 months after breast cancer operation. J Rehabil Med, 2005, Volume 37, pp.180-188.

[8] Binkley J, Stratford P, Gabram-Mendola S, Pearl M, et.al. Measurement of impairment and activity limitation in women following breast cancer surgery. Combined sections meeting of APTA, Feb 2011, New Orleans.

[9] Smoot J, Wong J, Cooper B, Wanek L, et.al. Upper extremity impairments in women with or without lymphedema following breast cancer treatment. J Cancer Surviv, 2010, Volume 4, Issue 2, pp. 167-178.

[10] Leidenius M, Leppanen E, Krogerus L, Smitten K. Motion restriction and axillary web syndrome after sentinel node biopsy and axillary clearance in breast cancer. Am J Surg, 2003, Volume 185, pp. 127-130.

[11] Fangel LMV, Panobianco MS, Kebbe LM, Almeida AM, et.al. Quality of life and daily activities performance after breast cancer treatment. Acta Paul Enferm, 2013, Volume 23, Issue 1, pp. 93-100.

\section{Author Profile}

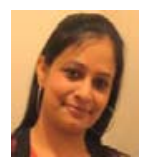

Prabhjot Kaur Gambhir, Maters of Occupational Therapy (Neurosciences) is an Occupational Therapist at Max Super Speciality Hospital, Saket, Delhi (India). Her field of work involves adult and paediatric neurorehabilitation including dysphagia management. She is certified in Vital Stim Therapy and conservative Dysphagia management as well as Breast Cancer rehabilitation. She has received a Service Excellence Award (GEM- Going an Extra Mile) during her work tenure at Max Hospital. She has international publications in dysphagia, neuro- rehabilitation and breast cancer rehabilitation. 